\title{
13. Vergleich und Tendenzen
}

\subsection{Allgemeines}

Im allgemeinen Vergleich kann man beobachten, dass sich der Fokus, nachdem strukturell bedingte Schwierigkeiten beseitigt waren, stark veränderte. So bauten nachfolgende ICOM-Präsidenten natürlich immer wieder auf der Vorarbeit ihrer Vorgänger auf. Dabei ist die Entwicklung jedoch weder stringent zu sehen, da jeder individuelle Schwerpunkte setzte, noch stagnierte sie oder war in irgendeiner Form rückschrittlich.

Während bei Prof. Günter Schade durch politische Umbrüche und der Vereinigung der beiden deutschen ICOM-Nationalkomitees an inhaltliche Verbandsarbeit kaum zu denken war, konnte sich Dr. Albert Treff einige Jahre darauf doch schon programmatischer am Geschehen beteiligen. Doch auch er hatte vordergründig strukturelle Verbandsangelegenheiten, wie die Vorbereitungen zum Aufbau einer Geschäftsstelle, zu bewältigen.

Die Amtszeit von Prof. Dr. Hans-Martin Hinz scheint jedoch besonders viele positive Entwicklungen mit sich gebracht zu haben. Nicht nur konnte unter ihm die ICOM-Geschäftsstelle in Berlin eingerichtet werden, er beschäftigte sich zudem mit den vielfältigsten museologischen Fragestellungen, national und international und zog damit die Aufmerksamkeit anderer ICOMNationalkomitees auf sich und das deutsche ICOM-Komitee. Sein besonderes internationales Engagement (beispielsweise auch mit CEICOM) verhalfen ihm nicht zuletzt $\mathrm{zu}$ weiteren Präsidentschaften sowohl auf europäischer ICOMEbene als auch im Weltverband.

Diese Fortschritte würdigend, baute Dr. York Langenstein mit seiner besonderen Leidenschaft für die Bekämpfung des illegalen Handels mit Kulturgütern darauf auf. Dieses und weitere museumsethische Themen rückten nun immer mehr in den Fokus, während die Bemühungen um CEICOM abnahmen, da man mit dem Ausgang zufrieden war. Nach vergleichsweise kurzer Amtszeit (Rücktritt aufgrund von gesundheitlichen Gründen) führte Dr. Klaus Weschenfelder die Reihe der Veröffentlichungen von Bänden zur Museumsethik sowie Tagungen zu diesen Themen fort. Doch auch er musste sich wieder mit vermehrt mit strukturellen Themen, genauer, Formalia der rechtlichen Rahmenbedingungen des Verbandes auseinandersetzen. Tagungen, Publikationen und Handreichungen, die während seiner Amtszeit durchgeführt und veröffentlicht wurden, sind unter dem Punkt Museumsethik zusammenzufassen. 
Dr. Michael Henker wiederum engagierte sich sehr in Bezug auf neue Verordnungen, Gesetzgebungen, aber auch sehr stark in der Provenienzforschung. Während seiner Amtszeit wurde auch dazu eine Publikation angestoßen. Frau Prof. Dr. Reifenscheid-Ronnisch ist die erste Frau an der deutschen ICOM-Spitze. Auch sie setzt ihre ganz eigenen Schwerpunkte. Auf aktuelle Themen wie Dekolonisierung oder auch den Umweltschutz legt sie besonders Wert. Zudem liegt ihr die Förderung des Museums-Nachwuchses sehr am Herzen.

Doch neben den einzelnen Schwerpunkten, die gesetzt wurden, durchzogen verschiedene Themen, manchmal mehr oder weniger, die Amtszeiten: die Verbandsstruktur, die es mit wachsenden Mitgliedszahlen immer wieder zu erweitern und erneuern galt, innen- oder außenpolitische Gegebenheiten oder etwa das Werben um die öffentliche Aufmerksamkeit, sowohl innerhalb als auch außerhalb des Fachpublikums in Deutschland.

\subsection{Tendenzen nach der Codierungsannäherung}

In vielen der Interviews sind trotz ganz individueller Herausforderungen und Gegebenheiten durch Aufbrechen des Textes und Abstrahieren anhand von Codes ähnliche Probleme und Herausforderungen während der Amtszeit der jeweiligen Präsidenten zu beobachten. Dieses Abstrahieren von Textbausteinen war ein wichtiger Bestandteil, um einzelne Aussagen in einem übergeordneten Themenkomplex sammeln und dadurch Gemeinsamkeiten oder auch herausarbeiten zu können. Dabei können sich Aussagen einzelner Codes bei der Zuordnung in Gruppen überschneiden, d.h. Mehrfachnennungen sind möglich. Wichtig sind bei der Identifizierung von Kernaussagen vorerst nicht die einzelnen, oft kleinteiligen Umstände, sondern diese in ein Gesamtbild einzuordnen, um allgemeiner gültige Aussagen treffen können. Wenn man nun weg von der Betrachtung der Interviews in ihrer Einzigartigkeit geht, stellt man fest, dass unabhängig von zeitlich bedingten oder soziokulturellen Herausforderungen ähnliche oder sogar gleiche Schwierigkeiten auftraten.

Ein Themenkomplex, der in verschiedensten Formen immer von Bedeutung war, waren die politischen Gegebenheiten oder Einflüsse. Auf nationaler Ebene waren dies neben politischen Rahmenbedingungen ${ }^{3 \mathrm{I}}$ vor allem finanzielle Zuwendungen aus öffentlicher Hand, die die Präsidenten beschäftigten.

31 Siehe Interviews Prof. Dr. Schade, Dr. Treff u. Prof. Dr. Hinz: Die Wiedervereinigung Deutschlands und der Fall des Eisernen Vorhangs waren eine gesellschaftliche Herausforderung, die auch die Museen und die Verbandsarbeit bei ICOM Deutschland betraf. 
Diese zu bekommen war oftmals zudem mit hohem bürokratischem Aufwand verbunden. ${ }^{32}$ Außerdem konnten neue Gesetzgebungen oder Empfehlungen die Arbeit beeinflussen. ${ }^{33}$ Oftmals war auch von öffentlicher Seite her die Expertise von Verbandsmitgliedern gefragt.

Dies impliziert, dass repräsentative Verbandsarbeit nur im Dialog und regem Austausch mit der Politik stattfinden kann. Die Rolle des Präsidenten von ICOM Deutschland als Teil eines Weltverbandes ist dahingehend nicht zu unterschätzen. Eine Zusammenarbeit mit der Politik ist also notwendig, die Gefahr aber auch nicht zu unterschätzen. ${ }^{34}$

Dies leitet einen weiteren Punkt ein, der die Arbeit der Präsidenten und der Präsidentin von ICOM maßgeblich beeinflusst. Man ist stets um Internationalität bemüht, um den Dialog, nicht nur zwischen Museumsdirektor*innen zweier Länder, sondern im Idealfall auch den Dialog zwischen Gesellschaften oder Gruppen herzustellen und zu intensivieren. Durch die im Dreijahresrhythmus getaktete Bodenseetagung steht das Deutsche Nationalkomitee in regem und vor allem regelmäßigen Austausch mit ICOM Österreich und ICOM Schweiz. Zusätzlich dazu finden neben den Jahrestagungen in Deutschland, Tagungen in Kooperation mit anderen Ländern auch im Ausland, statt. ${ }^{35}$ Die Kommunikation steht hierbei im Vordergrund.

Doch nicht nur die Kommunikation und der Austausch mit anderen ICOMKomitees war stets Thema, auch der Dialog mit der Öffentlichkeit wurde gesucht.

Die öffentliche Wahrnehmung des Verbandes stellte in Deutschland trotz kontinuierlich steigenden Mitgliedszahlen ein Manko dar. Trotz Verbesserungen wie stetige Anpassung und Erneuerung der Mitteilungen an die Mitglieder stellte offensichtlich nicht das gewünschte Ergebnis dar. ${ }^{36}$ Dies könnte wohl aber auch der rasanten Entwicklung neuer Technologien und Kommunikationsformen geschuldet sein. Der Verband muss sich selbst stets erneuern und auf den Prüfstand stellen, um mit aktuellen Entwicklungen Schritt zu halten.

Dialog ist im Allgemeinen zwar ein sehr weit gefasster Begriff, jedoch in der Verbandsarbeit von ICOM Deutschland von zentraler und vielschichtiger Bedeutung. Denn auch der Dialog zwischen der Vorstandsebene und den Ver-

32 Siehe Interview Dr. Weschenfelder S. $83 \mathrm{f}$.

33 Siehe Interview Dr. Henker S. 90 f.

34 Mit Blick auf die politische Situation in Russland, beschrieben von Dr. Henker S. 89 f.

35 Dies lässt sich in allen Interviews beobachten, angefangen von Prof. Dr. Günter Schade, der im Austausch mit anderen europäischen Ländern über die Neuerungen in Bezug auf den Fall der DDR beriet, bis hin zu aktuellsten Austauschprojekten unter der amtierenden Präsidentin Frau Prof. Dr. Reifenscheid.

36 Vgl. Interview Henker. S. 96. 
bandsmitgliedern sowie unter den Mitgliedern untereinander war ein wichtiges Thema. Netzwerke, die aus der Mitgliedschaft resultierten und auch resultieren sollten, fanden zum Wohlfallen der Präsidenten sowohl auf nationaler als auch internationaler Ebene statt ${ }^{37}$ und halfen mitunter bei der täglichen Museumsarbeit. Dieser Austausch ist nur einer der Bausteine zum gegenseitigen Verständnis der Kulturen und Länder, das ICOM proaktiv fördern möchte.

Doch die ehemaligen Präsidenten und die amtierende Präsidentin standen oder stehen nicht nur vor öffentlichkeitswirksamen Herausforderungen. Auch interne Verbandsstrukturen wollten stets erweitert und verbessert werden. Oft fehlten dazu schlichtweg die zeitlichen Kapazitäten, da der Vorsitz von ICOM Deutschland selbst »nur« ein Ehrenamt ist und von allen Interviewten neben deren hauptberuflichen Tätigkeiten ausgeübt wurde. So konnten die Präsidenten festhalten, dass der Erfolg von ICOM Deutschland auch einem funktionierenden Sekretariat mit den korrekten rechtlichen Grundlagen zu verdanken ist. $^{38}$

$\mathrm{Zu}$ beobachten ist neben den thematischen Schwerpunkten, dass wenig Kritik geäußert wurde. Antworten zum Verhältnis zur Geschäftsstelle in Paris bewegten sich oft nur an der Oberfläche.

$\mathrm{Ob}$ die Präsidenten selbst Versäumnisse während ihrer Amtszeit sahen, wurde oftmals mit der Aussage, dass man eben guten Gewissens getan hat, wofür neben dem Beruf noch Zeit blieb, beantwortet, beziehungsweise mit dem Aspekt des Ehrenamtes. ${ }^{39}$ Angesichts des hohen Zeitaufwandes und der großen Verantwortung, die solch ein Amt mit sich bringt, scheint dies legitim und aufrichtig.

37 Dr. Treff ging sogar so weit zu sagen, dass dies quasi die Hauptaufgabe des Verbandes sei. S. 47.

38 Vgl. Interview Weschenfelder S. 83 ff.

39 Vgl. Interview Langenstein S. 74 ff. 